\title{
Development of a kinematic simulation tool to study cylindrical plunge grinding
}

\author{
Patel, A. ${ }^{1}$, Tannahill, B. ${ }^{1}$, Bauer, R. ${ }^{1 *}$, Warkentin, A. ${ }^{1}$ \\ ${ }^{1}$ Department of Mechanical Engineering, Dalhousie University, Halifax, Nova Scotia, B3H 4R2, Canada \\ *robert.bauer@dal.ca
}

\begin{abstract}
This paper presents a novel cylindrical plunge grinding kinematic simulator which can be used to help predict the surface roughness of ground workpieces. To significantly reduce simulation time, an innovative linear interpolation approximation approach was used. For the conditions studied, this approach was found to reduce simulation time on subsequent wheel revolutions from $274 \mathrm{~s}$ per wheel revolution down to only $5.37 \times 10^{-4}$ s per wheel revolution - at the expense of increasing the errors in the resulting workpiece profiles by, on average, only $1.8 \mathrm{~nm}$. Experiments were then carried out for three different speed ratios $(4.41,4.59$ and 4.78$)$ to validate the simulator. It was found that there was excellent agreement between the experimental and simulated arithmetic mean workpiece surface roughness for the speed ratios tested.
\end{abstract}

Keywords-cylindrical, plunge, grinding, computer simulator, speed ratio

\section{INTRODUCTION}

In order to predict the workpiece surface finish in cylindrical plunge grinding a kinematic simulation approach similar to that used by Liu et al. [1] for point grinding was followed. To achieve the resulting workpiece surface, the individual trajectories of abrasive grains are simulated and any interactions between the abrasive grains and the workpiece are used to remove material from the workpiece model. As illustrated in Fig. 1, the cylindrical workpiece model consists of a 2D array of radials where the values in the array correspond to the heights of the workpiece surface at different

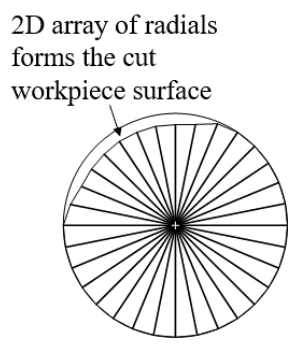

Figure 1. Cylindrical workpiece model

\footnotetext{
The authors would like to thank the Natural Sciences and Engineering Research Council of Canada (NSERC) and the Canadian Foundation for Innovation (CFI) for their financial support of this research.
}

locations around the workpiece to form the cut workpiece following a similar approach to the flat-grinding simulations developed by Chakrabarti and Paul [2] as well as Nguyen and Butler [3]. This paper extends these kinematic simulation approaches to cylindrical grinding for multiple grinding wheel revolutions and describes the development of a unique computationally-efficient cylindrical plunge grinding computer simulation tool using Matlab ${ }^{\mathrm{TM}}$ that can be used to help predict the resulting workpiece surface topology.

\section{SIMULATOR DEVELOPMENT}

For the 2D simulator, a rotating workpiece having nominal radius $R_{w}$ was used as a non-rotating frame of reference such that the grinding wheel of nominal radius $R_{S}$ appears to rotate around it as shown in Fig. 2a) and b). The fixed non-rotating base reference frame $\mathscr{T}_{0}$ has its origin at the center of the workpiece while Frame $\mathscr{1}$ has its origin at the wheel center and rotates with the grinding wheel. Referring to Fig. 2a), for a given depth of cut $a$, the distance between the workpiece and wheel center $r$ is given by:

$$
r=R_{s}+R_{w}-a .
$$

Based on the geometry shown in Fig. 2a) and b), the position of the wheel center relative to the workpiece center expressed in $\mathscr{F}_{0}$ as a function of time $P_{c 0}(t)$ can then be written as:

$$
P_{c 0}(t)=\left[\begin{array}{c}
r \cos \left(\frac{\pi}{2}-\omega_{w} t\right) \\
r \sin \left(\frac{\pi}{2}-\omega_{w} t\right) \\
0
\end{array}\right]
$$

where $\omega_{w}$ is the workpiece angular velocity and $t$ is time such that when $t=0 \mathrm{~s}$, the wheel center is directly above the workpiece center. For $t>0 \mathrm{~s}$, the wheel center follows a circular trajectory and appears to travel around the workpiece as shown in Fig. 2b).

As shown in Fig. 2c), there are $i$ abrasive grains which form cutting edges on the wheel. Using the geometry in Fig. $2 \mathrm{c})$, the $i^{\text {th }}$ cutting edge position relative to the wheel center expressed in $\mathscr{F}_{1}$ as a function of time $P_{i 1}(t)$ can be written in 
terms of angle $\theta_{s i}$, radius $R_{s i}$ and grinding wheel angular velocity $\omega_{s}$ as follows:

$$
P_{i 1}(t)=\left[\begin{array}{c}
R_{s i} \cos \left(\theta_{s i}+\omega_{s} t\right) \\
R_{s i} \sin \left(\theta_{s i}+\omega_{s} t\right) \\
0
\end{array}\right]
$$

such that the cutting edge corresponding to $i=1$ with $\theta_{s i}=0^{\circ}$ would be located at bottom-dead center on the grinding wheel.

Using (2) and (3), the corresponding location of the $i^{\text {th }}$ cutting-edge position relative to the workpiece center expressed in $\mathscr{T}_{0}$ as a function of time $P_{i 0}(t)$ can therefore be written as:

$$
P_{i 0}(t)=P_{c 0}(t)+R_{0}^{1}(t) P_{i 1}(t)
$$

where the rotation matrix $R_{0}^{1}$ which transforms the components of a vector expressed in $\mathscr{F}_{1}$ to the components of a vector expressed in $\mathscr{F}_{0}$ is defined as:

$$
R_{0}^{1}(t)=\left[\begin{array}{ccc}
\cos \left(-\omega_{w} t\right) & -\sin \left(-\omega_{w} t\right) & 0 \\
\sin \left(-\omega_{w} t\right) & \cos \left(-\omega_{w} t\right) & 0 \\
0 & 0 & 1
\end{array}\right]
$$

Substituting (1), (2), (3) and (5) into (4) yields the following expression for the position of the $i^{\text {th }}$ cutting edge as a function of time as shown in Fig. 2c):

$$
\begin{aligned}
& P_{i 0}(t)= \\
& {\left[\begin{array}{c}
r \sin \left(\omega_{w} t\right)+R_{s i} \cos \left(\theta_{s i}+\omega_{s} t+\omega_{w} t\right) \\
r \sin \left(\frac{\pi}{2}-\omega_{w} t\right)+R_{s i} \sin \left(\theta_{s i}+\omega_{s} t-\omega_{w} t\right) \\
0
\end{array}\right]}
\end{aligned}
$$

Fig. 3 illustrates the corresponding $i=1$ cutting edge motion over one revolution of the wheel for sample integer speed ratios $S=\omega_{s} / \omega_{w}=2,3$ and 4 , where $\omega_{s}$ and $\omega_{w}$ are the angular velocities of the wheel and workpiece, respectively. Note that the number of times each of the grain trajectories approaches the workpiece is equal to the speed ratio $S$ - a fact that was exploited to reduce computational time as will be discussed.

Fig. 4 illustrates how the cylindrical workpiece is modelled as equally-spaced radials. Increasing the number of radials effectively increases the resolution of the resulting ground workpiece surface. The grinding wheel cross section is modelled by setting the radius and angular spacing of the cutting edges around the wheel circumference.
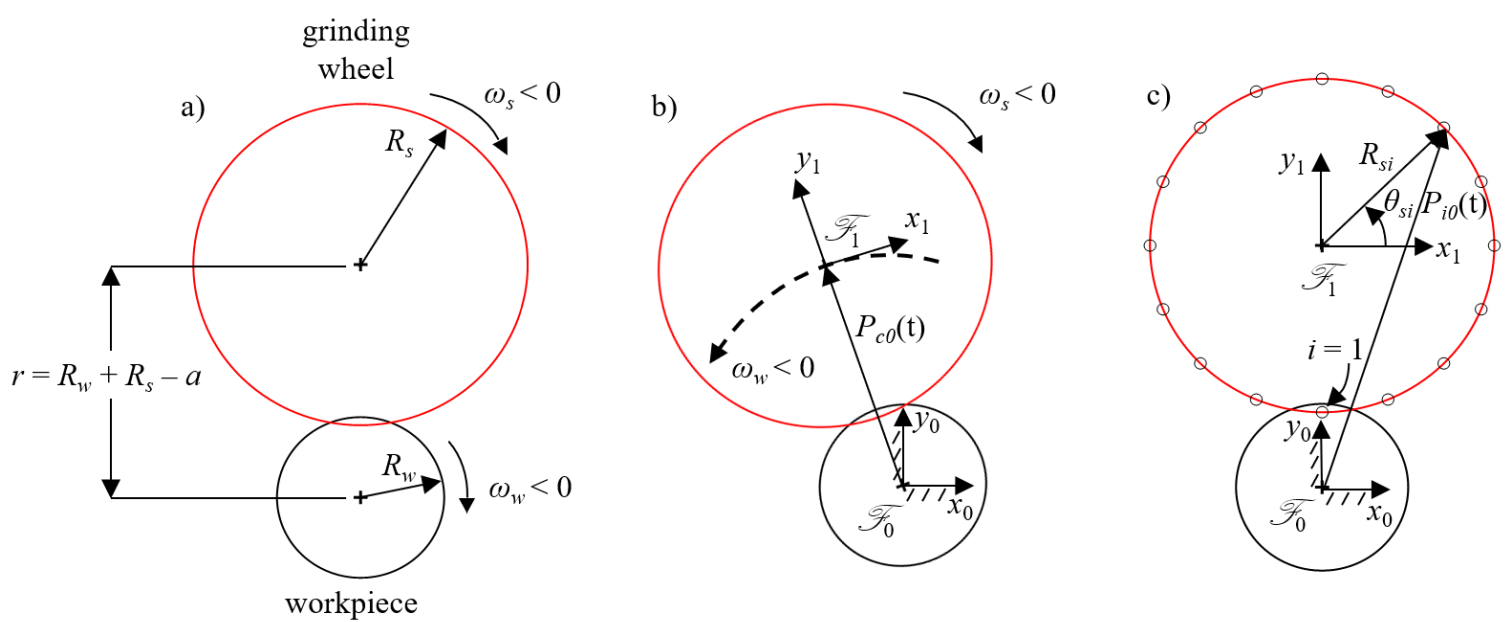

Figure. 2. Reference frames and nomenclature used in the cylindrical grinding computer simulator
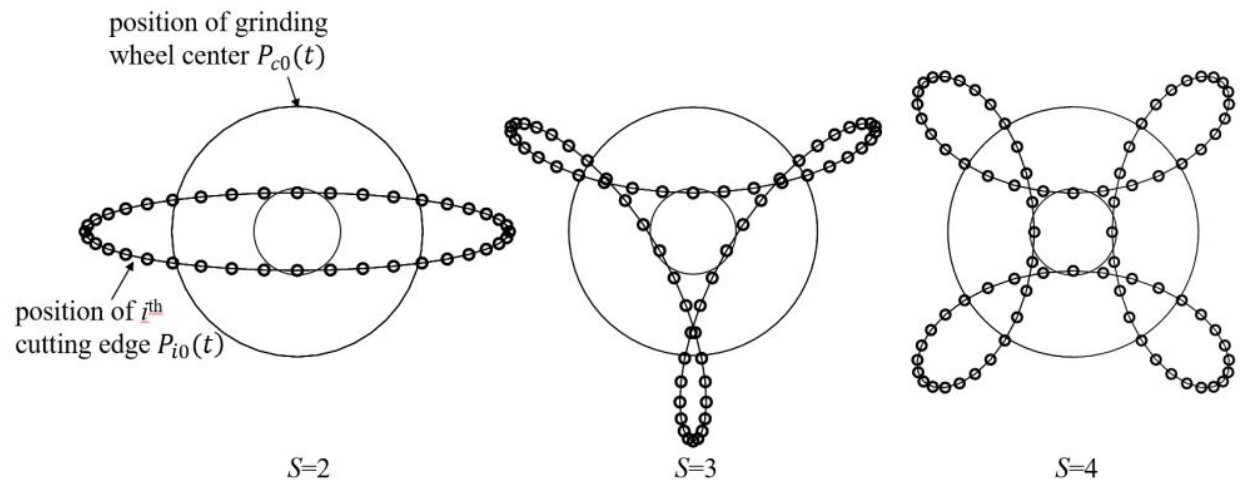

Figure 3. Sample $i=1$ grain trajectories for speed ratios $S=2,3$ and 4 


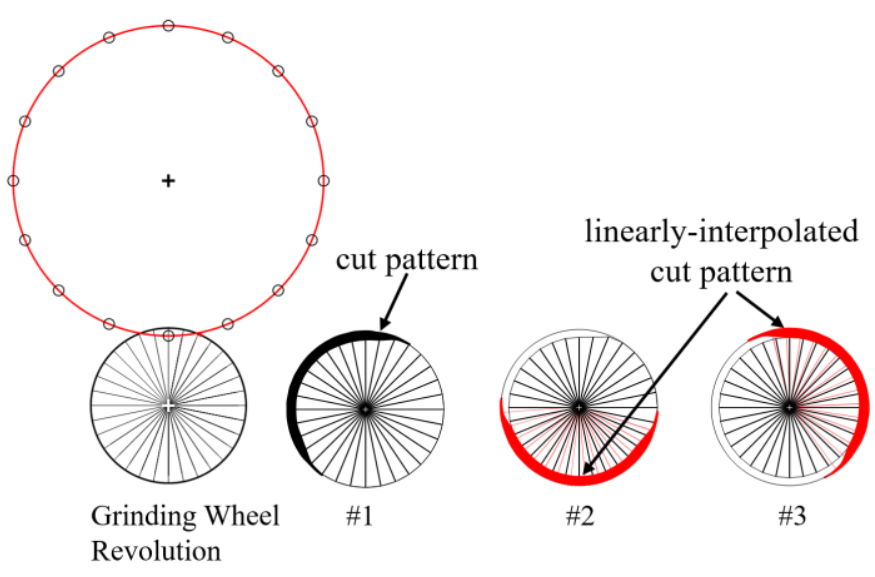

Figure 4. $S=3$, Wheel Rev. \#1: generate cut pattern on workpiece, Wheel Rev. \#2 and \#3: linear interpolation of cut pattern on workpiece radials

By tracking the trajectories of each cutting edge, the intersections of the grains with the workpiece radials can be calculated. This calculation was accomplished by first determining the times at which each cutting edge enters and exits the workpiece surface of radius $R_{w}$ for the first rotation of the grinding wheel only. These times were found by sequentially calculating the trajectory of each cutting edge over one grinding wheel revolution using a coarse time step to try to find a point on the trajectory that lies inside the workpiece's nominal diameter. The time step is iteratively reduced until at least one point on the trajectory lies within the workpiece. Knowing a time that the cutting edge lies inside the workpiece, and a time when the cutting edge has not yet penetrated the workpiece provides a bound on the desired time that the cutting edge enters the workpiece. A bounded-domain nonlinear search algorithm was then used with these bounds to numerically find the value of $t$ that minimize the following function:

$$
\min \left|\left\|P_{i 0}(t)\right\|_{2}-\left\|R_{w}\right\|_{2}\right|
$$

corresponding to the time at which the cutting edge enters the workpiece surface.

Similarly, the exit time can be found using (7) by setting the bounds on the solution to lie between the time a cutting edge lies inside the workpiece and a time when the cutting edge has left the workpiece. The cutting-edge entrance and exit times can then be used in conjunction with (6) to determine the angular location on the workpiece relative to $\%$ where the cutting edge enters and exits the workpiece surface and, therefore, which workpiece radials are potentially affected by the cutting edges. The entrance and exit times also provide a bound to increase the speed of the numerical solution for the time where the trajectory intersects each workpiece radial. This calculation can be accomplished for each affected radial by minimizing the following function:

$$
\min \left|\tan ^{-1}\left(\frac{y \text { component of } P_{i 0}(t)}{\mathrm{x} \text { component of } P_{i 0}(t)}\right)-\theta_{w k}\right|
$$

where $\theta_{w k}$ corresponds to the angle of the $k^{\text {th }}$ affected workpiece radial. Solving for the time at which each cutting edge intersects a workpiece radial enables the determination of how much each radial should be truncated to simulate material removal.

One of the most computationally-expensive parts of the simulation occurs when calculating the intersections of the cutting edges with the workpiece radials. To increase the speed of the simulator, the present authors propose to only calculate the radial cut pattern for one grinding wheel revolution. The authors then use linear interpolation to apply this radial cut pattern onto the radials that are affected by the second, third, fourth and $n^{\text {th }}$ grinding wheel revolutions. Using this unique approach, Fig. 4 illustrates the sequence of cuts a grinding wheel having sixteen equally-spaced grains makes on a workpiece having thirty-two radials using a sample speed ratio $S=3$. As shown in the figure, each revolution of the grinding wheel removes more workpiece material up to and including the third revolution, after which no additional workpiece material is removed because the cutting edge trajectory through the workpiece on the fourth grinding wheel revolution would follow the same path taken by the cutting edges on the first revolution of the wheel.

For a sample wheel having 4,080 cutting edges and a workpiece with 7,980 radials, this linear interpolation approach effectively reduces the compuational time for subsequent grinding wheel revolutions from 274 s per wheel revolution down to only $5.37 \times 10^{-4}$ s per wheel revolution. This time savings is particularly important since plunge grinding typically requires many grinding wheel revolutions during the grinding cycle. Fig. 5 plots a sample of the resulting workpiece surface profile errors that are incurred for each workpiec radial over one wheel revolution because of the linear interpolation approximation - which are, on average, only $1.8 \mathrm{~nm}$.

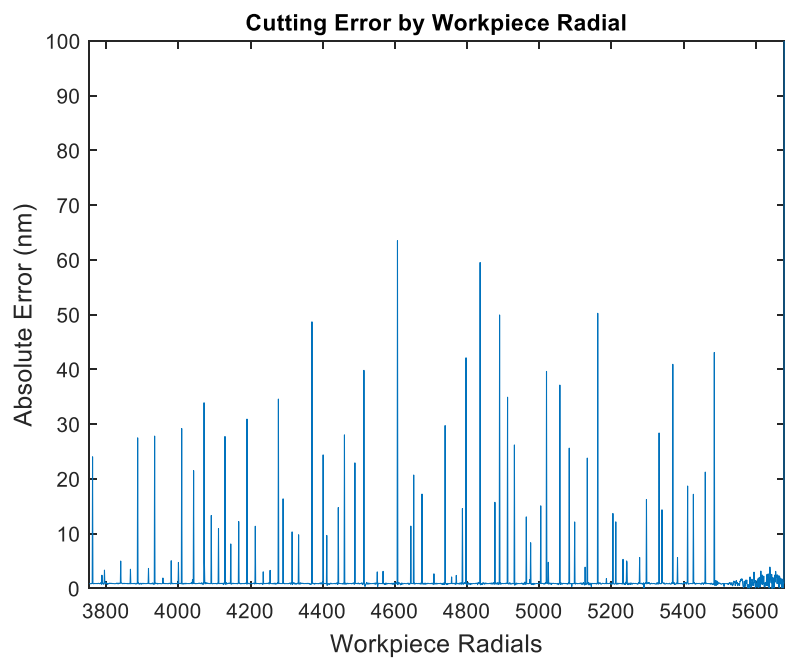

Figure 5. Workpiece surface linear interpolation errors for the workpiece radials affected by one revolution of the grinding wheel 


\section{EXPERIMENTAL VALIDATION}

To validate the workpiece surface predictions made by the simulator, a set of cylindrical plunge grinding experiments were carried out for sample speed ratios of $S=4.41,4.59$ and 4.78. Fig. 6 shows the experimental setup which uses a Blohm Planomat 408 grinding machine retrofitted with a custom rotary axis to enable cylindrical grinding. For these grinding experiments, a 0.4064m diameter Radiac Abrasives WRA60J5-W vitrified grinding wheel was dressed using a singlepoint diamond dresser having a width of $820 \mu \mathrm{m}$, an overlap ratio of 7.5 and depth of cut of $10 \mu \mathrm{m}$. The grinding experiments used a wheel speed of 1,000rpm to grind a $0.254 \mathrm{~m}$ diameter precision ground AISI 1045 steel workpiece with a dwell time of 10 seconds. A plunge infeed rate of $1.27 \mu \mathrm{m} / \mathrm{rev}$ was used up to a depth of cut of $76 \mu \mathrm{m}$ - for a total of about 227 grinding wheel revolutions for a single plunge grinding cycle. CIMTECH310 coolant was applied via a coherent jet having a concentration of $5.1 \%$ and a flowrate of $381 \mathrm{pm}$.

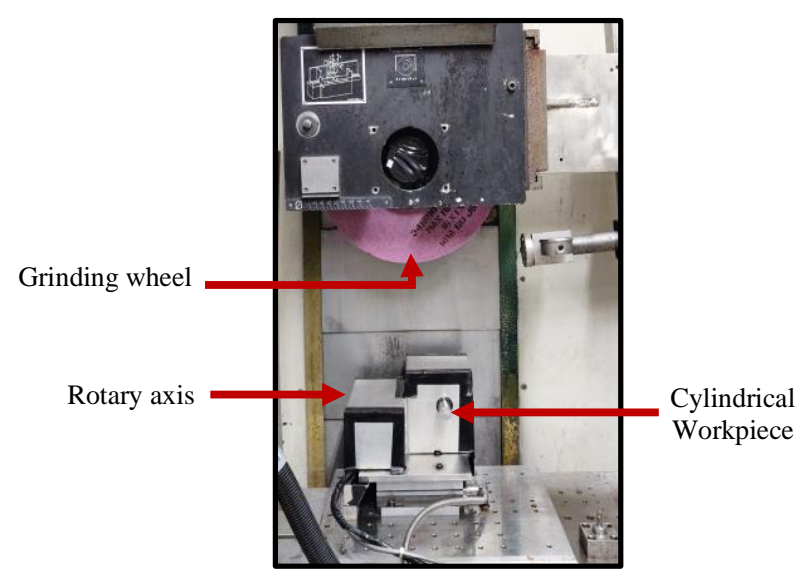

Figure 6. Experimental setup

To calculate the resulting arithmetic mean workpiece surface roughness $R_{a}, 2.5 \mathrm{~mm}$ profiles were measured at three random locations around the workpiece's circumference using a non-contact Nanovea profilometer. Experiments were carried out twice to ensure repeatability of the results and the measured six $\mathrm{R}_{\mathrm{a}}$ values were averaged for each speed ratio.

The development of the grinding wheel model used in the simulator was based on the approach used by Darafon et al. [4] for flat grinding. The nominal grain diameter $d_{g}$ was determined from the grinding wheel grit number $M=60$ as follows [5]:

$$
d_{g}=15.2 M^{-1}=\frac{15.2}{60} \mathrm{~mm}
$$

while the grain volume fraction $V_{g}$ was calculated with the wheel's structure number $S_{\text {struct }}=5$ by [5]:

$$
V_{g}=0.02\left(32-S_{\text {struct }}\right)=0.02(32-5)
$$

Applying the torus method, the number of grains on the wheel circumference $n_{g}$ can then be approximated as described in [4] using:

$$
n_{g}=\frac{3}{2} \pi V_{g}\left(\frac{d_{s}}{d_{g}}-1\right)=4,080
$$

where $d_{g}=0.4064 \mathrm{~m}$ is the nominal wheel diameter. The resulting 4,080 grains were initially evenly distributed around the grinding wheel after which the protrusion heights and spacing were adjusted using a uniform random distribution. The random protrusion height pattern was then tuned to minimize the predicted error in surface roughness compared with the experimental measurements. The workpiece was modelled using 7,980 radials corresponding to a circumferential resolution of $0.1 \mu \mathrm{m}$. Note that the simulation was run for the $10 \mathrm{sec}$ spark out portion of the grinding cycle that corresponds to about 167 grinding wheel revolutions. The interpolation process reduced the resulting simulation time from about 12.7 hours to only 4.6 minutes.

Fig. 7 shows a comparison of the experimental and simulated workpiece surface roughness $\mathrm{R}_{\mathrm{a}}$ for the three speed ratios tested with experimental error bars corresponding to three standard deviations. It can be seen in Fig. 7 that the workpiece surface roughness predicted by the simulator agreed with the experimentally-measured values for the three speed ratios tested. The average difference in $R_{a}$ values between experimental and simulated workpiece surface roughness was only about $1.5 \%$.

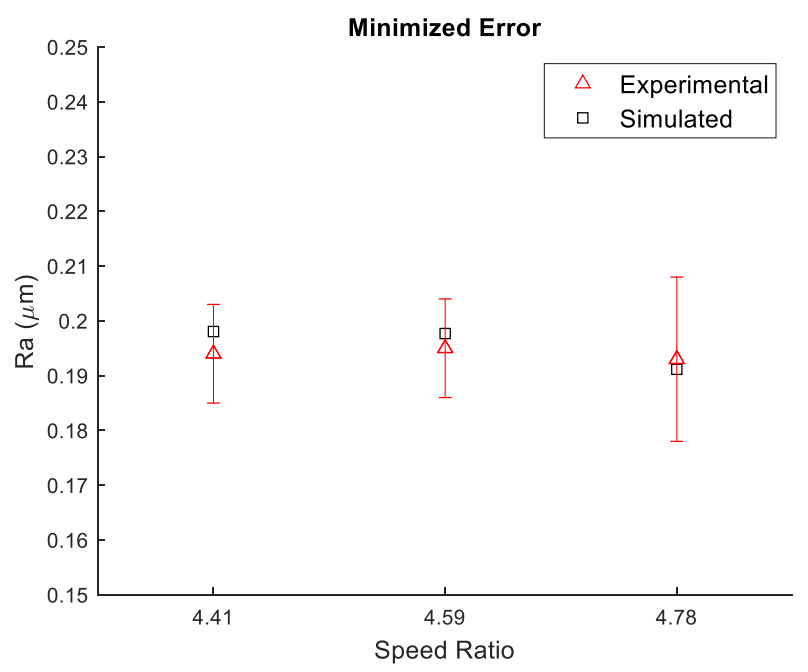

Figure 7. Comparison of experimental and simulated workpiece $\mathrm{R}_{\mathrm{a}}$ for $S=4.41,4.59$ and 4.78

\section{SUMMARY}

The cylindrical plunge grinding simulator described in this paper successfully incorporated a novel interpolation approach such that the predicted cut pattern on the workpiece from the first grinding wheel revolution could be linearly interpolated onto the workpiece for subsequent wheel rotations. For the conditions used in this research, this feature of the simulator was able to reduce computation time from 
274 s per wheel revolution down to only $5.37 \times 10^{-4}$ s per wheel revolution, while incurring average workpiece profile prediction errors of only $1.8 \mathrm{~nm}$ when using a wheel model with 4,080 grains and a workpiece circumferential resolution of $0.1 \mu \mathrm{m}$. In this research, a single plunge grinding cycle having a depth of cut of $76 \mu \mathrm{m}$ required $\sim 227$ grinding wheel revolutions. Using the proposed interpolation approach to simulate this plunge grinding cycle would reduce simulation time from over 17 hours down to only 4.6 minutes. This significant reduction in simulation time makes it much more computationally feasible to test myriad grinding process parameters for sensitivity and optimization studies, and could potentially lead to more computationally-efficient 3D grinding simulators.

Experiments were then conducted for sample speed ratios of $S=4.41,4.59$ and 4.78. When compared with experiments, the workpiece surface roughness predicted by the simulator agreed very closely for the speed ratios tested. It may, therefore, be possible to use the simulator to help determine optimal speed ratios in cylindrical plunge grinding.

\section{REFERENCES}

[1] Y. Liu, Y. Gong, R. Bauer, and A. Warkentin, "Experimental and numerical investigation into workpiece surface topology in point grinding," Proc. IMechE. Part B: J. Eng. Manuf., 2012, vol. 226, pp. $1793-1800$.

[2] S. Chakrabarti, S. Paul, "Numerical modelling of surface topography in superabrasive grinding," Int. J. Adv. Manuf. Technol., 2008, vol. 39, pp. 29-38.

[3] T.A. Nguyen, D.L. Butler, "Simulation of surface grinding process, part 2: interaction of the abrasive grain with the workpiece," Int. J. Mach. Tool. Manuf., 2005, vol. 45, pp. 1329-1336.

[4] A. Darafon, A. Warkentin, R. Bauer, "3D metal removal simulation to determine uncut chip thickness, contact length, and surface finish in grinding,” Int. J. Adv. Manuf. Technol., 2013, vol. 66, pp. 1715-1724.

[5] S. Malkin, "Grinding technology: theory and applications of machining with abrasives," Dearborn, Michigan: Society of Manufacturing Engineers, 1989. 